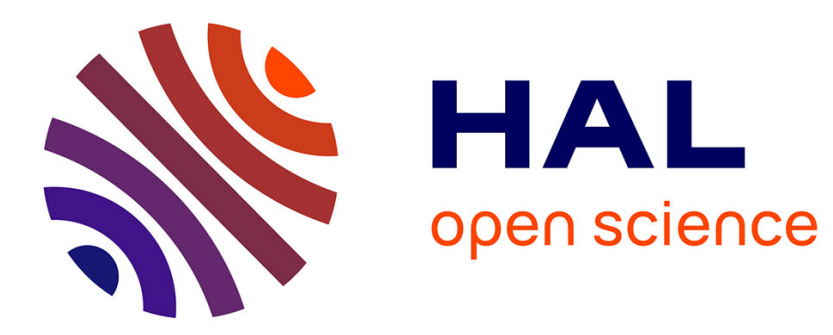

\title{
Étude sémantique et syntaxique de ÊTRE EN TRAIN DE
}

\author{
Danh Thành Do-Hurinville
}

\section{To cite this version:}

Danh Thành Do-Hurinville. Étude sémantique et syntaxique de ÊTRE EN TRAIN DE. L'information grammaticale, 2007, 113, pp.32-39. hal-00497581

\section{HAL Id: hal-00497581 \\ https://hal.science/hal-00497581}

Submitted on 5 Jul 2010

HAL is a multi-disciplinary open access archive for the deposit and dissemination of scientific research documents, whether they are published or not. The documents may come from teaching and research institutions in France or abroad, or from public or private research centers.
L'archive ouverte pluridisciplinaire HAL, est destinée au dépôt et à la diffusion de documents scientifiques de niveau recherche, publiés ou non, émanant des établissements d'enseignement et de recherche français ou étrangers, des laboratoires publics ou privés. 


\title{
Étude sémantique et syntaxique de ETTRE EN TRAIN DE
}

\author{
Danh Thành DO-HURINVILLE \\ MoDyCo, Université Paris Ouest (Nanterre), UMR 7114 \\ Institut National des Langues et Civilisations Orientales
}

L'évolution sémantique de la périphrase être en train de dans Je suis tellement en train de suer que je sue toujours (Sévigné, 1735, Littré : 6413) et Puisque je suis en train de soleils couchants, de crépuscules et de clairs de lune, il faut que je vous raconte ma soirée d'avant-hier (Hugo, Rhin, 1842, TLF ${ }^{1}$ : 461), par rapport à celle utilisée de nos jours dans Je suis en train de travailler, nous a conduit à la rédaction de cet article, composé de trois parties.

Nous procéderons d'abord, d'une part à une description de train, être en train et être en train $d e$, d'autre part à la recherche de l'invariant sémantique entre train et être en train de, et à une évaluation du degré de grammaticalisation de cette périphrase ; puis à une analyse sémantique et syntaxique de être en train de avec les quatre types de procès (activités, accomplissements, achèvements, états), avec déjà, encore, toujours, en ce moment, et avec les formes simples et les formes composées du mode indicatif.

\section{TRAIN, ETRE EN TRAIN, ETRE EN TRAIN DE}

\subsection{Le substantif train et quelques locutions dérivées}

Selon le Dictionnaire historique de la langue français (DHLF : 2148), le verbe traîner, issu du latin populaire traginare, lui-même dérivé du type assez rare tragere, forme altérée de trahere «tirer », qui a donné traire, a produit les déverbaux train et traîne.

Le substantif train, qui a connu un développement sémantique très riche étendu sur plusieurs siècles, désigne d'abord (fin XII ${ }^{\mathrm{e}}$ siècle) un ensemble de choses, et dans les chansons de geste, de cadavres dont la terre est jonchée ; cette valeur est propre à l'ancien français. Ses autres sens anciens, "convoi de bêtes voyageant ensemble " (fin XII ${ }^{\mathrm{e}}$ siècle), "file de bêtes de somme voyageant ensemble avec le personnel de service» (milieu XIII ${ }^{\mathrm{e}}$ siècle), et «ensemble de domestiques, de chevaux, de voitures accompagnant une personne (milieu XIII ${ }^{\mathrm{e}}$ siècle), comme dans un train de mulets portant des vivres, qui ne sont plus usités, ont donné naissance à plusieurs sens nouveaux soulignant l'idée de file, de suite en mouvement: «allure, mouvement » du cheval ou d'une monture : chevaux qui accélèrent leur train ( $\mathrm{XV}^{\mathrm{e}}$ siècle); «manière d'aller, marche des choses »: Voilà le train du monde (Montaigne, fin $\mathrm{XVI}^{\mathrm{e}}$ siècle) ; «allure très rapide, excessive »: galoper, rouler à un train d'enfer, mener un train d'enfer; «allure lente, grave et majestueuse »: Train de sénateur, etc. Au début du XIX siècle, une extension concrète de l'idée de « suite, convoi, file » a engendré le sens de « suite de voitures ou de wagons attelés les uns aux autres et traînés sur des rails par une locomotive » : train de chariots, puis par métonymie celui de «voyageurs d'un train»: train de voyageurs. Les expressions prendre le train, manquer le train viennent de ce sens.

1 Sigles des dictionnaires consultés : TLF (Trésor de la Langue Française, tome 16, 1994), DHLF (Dictionnaire Historique de la Langue Française, 1992), GRLF (Le Grand Robert de la Langue Française, 1985), Littré, Dictionnaire de la Langue Française (tome 6, 1994). 


\subsection{La périphrase être en train}

Selon le $D H L F$ (ibid.), dès le XVI ${ }^{\mathrm{e}}$ siècle, train, combiné à la préposition en et au verbe être, forme la périphrase être en train $^{2}$ signifiant « en action, en mouvement, en cours d'exécution, ou en bonne disposition physique ». On rencontre (être) en train dans des phrases dont le sujet est une personne: Puisque vous êtes en train, vous connaîtrez par là les sentiments que votre famille a pour vous (Molière, Le Malade imaginaire, 1673, Fournier, 1998 : 254). Personne ne se sentait en train, ni les maîtres, ni les élèves (Daudet, Le Petit Chose, 1868, GRLF : 414). Dans des phrases dont le sujet désigne un inanimé, être en train veut dire «être commencé, en bonne voie d'exécution, en cours » : J'ai une grosse commande en train avec la compagnie du gaz (Cendrars, Bourlinguer, 1948, TLF : 461).

On citera aussi mettre en train, dont l'objet peut désigner aussi bien une personne qu'un inanimé. Mettre quelqu'un en train signifie «s'exciter à la joie, au plaisir » : Pour se mettre en train, il versa une bonne rasade (Dabit, Hôtel Nord, 1929, TLF : 461). Quant à mettre quelque chose en train $^{3}$, elle signifie «commencer l'exécution, l'utilisation de quelque chose »: Puis-je compter sur vous pour mettre cela en train et établir des liaisons entre les comités et nous (De Gaulle, Mémoire de guerre, 1954, TLF: 461). L'expression mise en train de quelque chose (mise en train d'une affaire, d'une production) indique les travaux de préparation qui la mettent en marche et précèdent la période de plein rendement (GRLF : 414).

\subsection{La périphrase être en train de}

Dans son emploi ancien $\left(\mathrm{XVI}^{\mathrm{e}}\right.$ siècle), être en train de signifiant «être dans une disposition de, en humeur de, disposé à » peut se combiner aussi bien avec un substantif (être en train de confidence, d'énergie, d'optimisme, TLF : 461) ${ }^{4}$, qu'avec un verbe à l'infinitif : Savez-vous qu'il n'est pas bien de mettre les gens en train de nous aimer et de les planter là ? (Sénac de Meilhan, Emigré, 1797, TLF : 461); Le soir, promené à la jetée, pour laquelle je reprends du goût. J'étais en train d'être seul et n'ai point été chercher Chenavard (Delacroix, Journal, 1854, TLF : 461) $)^{5}$.

Gougenheim (1929 : 62-64) met l'accent sur la lente reconnaissance de l'emploi aspectuel de être en train de + infinitif par le dictionnaire de l'Académie. Ce n'est qu'à partir du milieu du $\mathrm{XIX}^{\mathrm{e}}$ siècle que cette périphrase sera interprétée comme traduisant l'aspect progressif ${ }^{6}$. Selon l'auteur, il est parfois difficile, entre le XVIII siècle et le début du XIX ${ }^{\mathrm{e}}$ siècle, de donner une interprétation exacte à être en train de, en soulignant que, dans Enfin, te voilà en train de faire ta fortune (Le Sage, Turcaret, 1709, Gougenheim : 63), cette périphrase n'a pas encore « le sens actuel, purement duratif, mais le sens ancien est loin déjà », et que dans Comme je suis en train de vous rendre compte de tout, il faut vous dire que ce Demoulin, qui voulait faire imprimer vos lettres, est celui qui suscita l'infâme procès de Jore (1737, Gougenheim : 63), cette expression signifie plutôt «puisque je suis en disposition de, puisque je suis entraîné à... » ${ }^{7}$.

Le tableau suivant résume l'évolution sémantique de être en train de, qui ne vient pas directement du sens propre du substantif train, mais de son sens figuré :

2 Il convient de distinguer en train en deux mots et entrain en un mot. Ce dernier est un nom masculin, dérivé probablement du verbe entraîner (XII ${ }^{\mathrm{e}}$ siècle), lui-même issu du verbe traîner, préfixé par en-.

3 Mettre quelque chose en train équivaut à mettre qqch. en chantier (ou sur le chantier), ou à mettre qqch. en route. La mise en route d'une affaire correspond à la mise en train d'une affaire.

4 Voir la phrase de Hugo, citée dans l'Introduction.

5 Voir la phrase de Sévigné, citée dans l'Introduction : en train de suer signifie « en disposition de suer ».

6 Comme Mortier (2004), nous préférons le terme progressif à duratif, utilisé par les dictionnaires consultés et adopté par Gougenheim (op. cit. : 62) : «Mais la véritable périphrase durative en français moderne, d'origine relativement récente, est en train de ».

7 Gougenheim (op. cit. : 64, note 62) signale que dans le parler actuel des Canadiens français, être en train de peut signifier « être occupé à »: La maman est en train de coucher ses enfants. Elle est en train de coudre. 


\begin{tabular}{|c|c|}
\hline (XII-XIII' s.) & Train « équipage, convoi, suite... » \\
\hline$\left(\mathrm{XV}-\mathrm{XVI}^{\mathrm{e}} \mathrm{s}.\right)$ & Train « allure, mouvement » \\
\hline \multirow[b]{2}{*}{$\left(\mathrm{XVI} \mathrm{e}^{\mathrm{e}} \mathrm{s}.\right)$} & $\begin{array}{l}\hat{E} \text { tre en train « en action, en mouvement, en humeur d'agir » } \\
\text { Mettre quelqu'un en train « le disposer à agir, l'inciter à la gaieté » } \\
\text { Mettre quelque chose en train « commencer l'exécution » }\end{array}$ \\
\hline & $\begin{array}{c}\text { Etre en train de + infinitif / ou substantif } \\
\text { « être en disposition de, être en humeur de, être disposé à » }\end{array}$ \\
\hline (depuis milieu $\mathrm{XIX}^{\mathrm{e}} \mathrm{s}$.) & $\begin{array}{c}\hat{E} \text { tre en train de }+ \text { infinitif « déroulement d'une action en cours » } \\
\text { (aspect progressif) }\end{array}$ \\
\hline
\end{tabular}

\subsection{A la recherche d'un invariant sémantique (train, en train, en train de)}

Par métaphorisation, le substantif train a perdu son sens initial pour donner progressivement naissance à différents sens figurés. Ayant opté pour le point de vue monosémique, nous chercherons un invariant sémantique inhérent au mot train ainsi qu'à ses locutions dérivées, et nous noterons que les acceptions « mouvement, action, allure », très proches les unes des autres, représentent une scène de déplacement de bêtes de somme, de chevaux et de voitures partageant les traits sémantiques [- humain / + dynamique], qui permettent de décrire, de façon figurée, l'état d'une personne [+ humain / + dynamique], qui est en action, en mouvement, en humeur d'agir, ou en bonnes dispositions physiques pour faire quelque chose. Etre en train de, qui a une valeur «plutôt modale qu'aspectuelle» (Pusch, 2003: 502), a subi, par la suite, « une démodalisation » pour n'indiquer que l'aspect progressif.

Le trait [+ dynamique] est donc l'invariant sémantique de train et de être en train de, ce qui explique pourquoi, de nos jours, cette périphrase est compatible avec les procès dynamiques, alors qu'elle accepte difficilement les procès non dynamiques.

\subsection{Evolution sémantique de être en train de et son degré de grammaticalisation}

\section{a. Evolution sémantique}

L'évolution de train vers être en train de semble conforme aux «chemins sémantiques », présentés par Marchello-Nizia (2006: 117) : mouvement > aspect ou auxiliaire modal > auxiliaire aspectuel, en ce sens que train, qui exprime un mouvement, forme être en train de, marquant l'aspect progressif, et que être en train de, au sens modal de «en disposition de », devient ensuite un auxiliaire aspectuel tel qu'on le connaît actuellement.

A l'examen de ses emplois anciens et actuels, nous notons que la périphrase en train de peut saisir le procès en trois phases (antérieure, initiale, médiane) comme suit :

- Première phase (ou phase antérieure $)^{8}$ : en train de exprimait «l'imminence de l'exécution d'une action» (fin XVII ${ }^{\mathrm{e}}$ siècle, DHLF : 2148), correspondant alors à l'aspect imminentiel. L'extrait suivant (Sévigné, 1681, GRLF : 414) : «[...] N'avez-vous pas de l'étoffe pour présenter au Roi ? Votre nom (de Grignan) est-il barbare ? N'est-il point en train de vous faire du bien? » montre que en train de équivaut à en passe de, en voie de.

- Deuxième phase (ou phase initiale) : en train de traduit l'aspect inchoatif (ou ingressif), comme l'illustrent les exemples suivants : selon Fuchs et Léonard (1979: 331), «Jean est en train d'aimer Marie » peut être paraphrasé de la façon suivante : "Jean commence à trouver Marie aimable » ou « Marie a su se rendre aimable ». Quant à Leeman (2003 : 28$29)$, elle estime que «Les enfants sont en train de manger» peut signifier « enfin! ils se mettent à manger », et que cet énoncé «ne suppose pas en soi qu'elle (= l'action) va se poursuivre contrairement à ce qu'indiquent certains dictionnaires ». Nous constatons que

8 Selon Cosériu (1980: 22), un procès verbal peut être observé avec cinq phases : avant le procès (phase antérieure), début du procès (phase initiale), déroulement du procès (phase médiane), fin du procès (phase finale), après le procès (phase postérieure). On attribue à ces phases respectivement les cinq aspects suivants : l'aspect imminentiel, l'aspect ingressif, l'aspect progressif, l'aspect conclusif, et l'aspect égressif. 
les gloses de ces énoncés mettent l'accent sur l'aspect inchoatif indiqué par « commencer à », ou « se mettre à », ou le verbe « savoir » au passé composé.

- Troisième phase (ou phase médiane) : en train de, exprimant l'aspect progressif, désigne le moment le plus intense du procès, au sens de in the midst of (cf. C. Smith, 1991 : 262) : «Elle est en train de balayer la chambre d'amis » (She is in the midst of sweeping the guest room). L'expression in the midst of signifie « au milieu de », répondant exactement à l'étymologie de en plein, ce qui explique pourquoi, dans certaines situations, en train de peut commuter avec en plein.

\section{b. Degré de grammaticalisation}

Le français actuel, à la différence de l'anglais, ne dispose pas de morphologie flexionnelle exprimant l'aspect progressif, alors qu'il avait autrefois être + PP (cf. Posner, 1998 : 104). Selon Pusch (2003 : 503), «le fait que l'aspect progressif s'exprime, en français comme dans les autres langues romanes, à travers de constructions périphrastiques et non pas par une morphologie flexionnelle constitue en soi un signe de grammaticalisation réduite ». Quant à Gougenheim (op. cit. : 65), il pense que « la locution est donc actuellement complètement entrée dans l'usage. Le mot train a perdu toute espèce de valeur propre et est devenu un simple morphème indiquant l'aspect duratif ».

Nous constatons en effet un processus de grammaticalisation, unidirectionnel, du lexème train vers la forme grammaticale : être en train de. Ce phénomène souligné par Meillet (1912: 132) : «l'attribution progressive d'un rôle grammatical à des mots autonomes... » existe probablement dans toutes les langues ${ }^{9}$. La grammaticalisation de cette périphrase française est achevée : celle-ci n'est plus suivie de noms, n'est compatible qu'avec des verbes, et ne traduit plus que le sens aspectuel progressif. Nous pensons qu'elle a atteint le dernier stade, intitulé "conventionalisation ${ }^{10}$, car train dans être en train de a perdu son sens lexical, et il peut coexister sans aucune ambiguïté avec le substantif train dans un même énoncé : Le train est en train de partir $^{11}$, selon le « principe d'anachronie » développé par Hagège (1993).

\section{ANALYSE SEMANTIQUE ET SYNTAXIQUE DE ETRE EN TRAIN DE}

\subsection{Etre en train de avec les quatre types de procès}

Nous appuyant sur les travaux menés à la suite de Vendler (1967), par Martin (1988), Fuchs et al. (1991), et Gosselin (1996), nous étudierons successivement être en train de avec les quatre classes de verbes suivantes (Gosselin, op. cit. : 41) :

- les verbes d'activité, qui sont dynamiques [+ dynamique], mais non bornés [- borné], ni ponctuels [- ponctuel] : marcher, manger, courir, etc.

- les verbes d'accomplissement, qui sont dynamiques [+ dynamique], bornés [+ borné], mais non ponctuels [- ponctuel] : courir un cent mètres, dessiner un cercle, etc.

- les verbes d'achèvement, qui sont dynamiques [+ dynamique], bornés [+ borné], et ponctuels [+ ponctuel] : apercevoir un avion, atteindre un sommet, etc.

- les verbes d'état, qui ne sont ni dynamiques [- dynamique], ni bornés [- borné], ni ponctuels [- ponctuel] : être fatigué, être gentil, savoir l'anglais, aimer la musique, etc.

En considération de leurs traits sémantiques ci-dessus, on oppose les verbes dynamiques (activités, accomplissements et achèvements) aux verbes non dynamiques (états).

9 Notons qu'en vietnamien, langue isolante, le mot dang, d'origine verbale, est grammaticalisé pour devenir un coverbe d'aspect. L'approche traditionnelle le considère comme un coverbe de temps exprimant le présent.

10 Selon le modèle quadriphasé de Heine (2002, cité par Marchello-Nizia, 2006 : 258) : le stade I, stade initial (initial stage), stade II, contexte de transition (bridging context), stade III, contexte de passage (switch context), et stade IV, conventionalisation.

11 Il en va de même pour beaucoup de beaux coups d'épée ou Il va aller au marché (Marchello-Nizia, 2006 : 261). 
Dénotant le trait [+ dynamique], la périphrase être en train de est considérée comme un « indicateur de processus » (Desclés, 1991 : 181). Lorsqu'elle est au présent ou à l'imparfait, elle parait fiable pour tester la dynamicité (Gosselin, op. cit. : 44). Elle doit donc être compatible avec les classes verbales pourvues de ce trait, à savoir les activités et les accomplissements qui sont des «processus », selon le classement de Desclés (op. cit.).

\section{a. Les activités}

Selon Gosselin (op. cit. : 54), une activité est une « série de changements prise comme stable, comme une situation ayant un début et une fin, sans que ce début et cette fin soient envisagés comme impliqués par le procès ». Lorsque la périphrase être en train de se combine avec un procès de type activité, elle indique le déroulement du procès par rapport à un point de référence choisi, et présuppose que ce procès prendra fin à un moment donné, après ce point de référence. Examinons les quelques situations suivantes :

(1) Paul est en train de travailler (chanter, dormir).

(2) Le capitalisme est en train de s'autodétruire (titre d'un livre de Artus et Virard).

Dans (1), dont le point de référence est simultané au moment de l'énonciation $t^{\circ}$, les procès travailler (chanter, dormir) avec être en train de se déroulent actuellement à $\mathrm{t}^{\circ}$, et seront achevés en principe à un moment donné après $t^{\circ}$. Cette périphrase peut indiquer une situation dont la fin est proche de $\mathrm{t}^{\circ}$ dans (1), ou éloignée de $\mathrm{t}^{\circ}$ dans (2).

(3) ?? La terre est en train de tourner autour du soleil ${ }^{12}$.

Bien que le procès tourner soit une activité, l'emploi de être en train de ne nous semble pas possible dans (3), qui décrit une vérité générale. Toutefois, on peut recourir à cet énoncé dans le cas où le locuteur, pour faire une démonstration, décrit le mouvement de la terre dans un cours de physique, par exemple.

(4) a. ? Il est en train de bien lire.

b. Il est en train de lire de travers (Franckel, 1989: 78).

(5) a. ? Il est en train de mettre sa culotte à l'endroit.

b. $\quad$ Il est en train de mettre sa culotte à l'envers (Franckel, ibid.).

Selon Franckel, les énoncés qui tendent à marquer « une conformité » dans (4a et 5a) sont moins tolérés que ( $4 b$ et $5 b$ ) qui marquent «une discordance ou une non-conformité ». Nous pensons que (4a et 5a) ne sont pas impossibles, mais peut-être s'en sert-on moins que de (4b et $5 b)$, car en général, les cas négatifs suscitent plus de problèmes.

(6) a. Ne sois pas toujours en train de râler (grogner, gueuler, pester, geindre, maugréer, hurler, se plaindre...).

b. ? Ne sois pas toujours en train de parler gentiment.

(7) a. ?? Sois toujours en train de râler (grogner, pester...)

b. ?? Sois toujours en train de parler gentiment.

L'énoncé impératif (6a) à forme négative et à valeur dépréciative nous semble beaucoup plus naturel que (6b) à valeur appréciative. L'énoncé (6a) est donc considéré comme un reproche fait par le locuteur à son allocutaire. Les énoncés assertifs correspondants (7a et $7 \mathrm{~b}$ ) ne sont acceptables, ni pour une valeur appréciative, ni pour une valeur dépréciative.

\section{b. Les accomplissements}

Un accomplissement est une «série de changements prise comme stable, mais dont le début et la fin sont perçus comme intrinsèques au procès lui-même » (Gosselin, op. cit. : 55).

(8) Paul est en train de dessiner un oiseau (courir un cent mètres, chanter une chanson, boire un verre d'eau, fumer une cigarette, manger un gâteau, écrire une lettre, etc.).

12 «a composition être en train de + infinitif interdit au présent et à l'imparfait toute acception gnomique ou habituelle » (Wilmet, $1980: 59)$. 
Notons que dessiner, courir, chanter, boire, fumer, manger, écrire sont autant d'activités : le début et la fin de ces procès ne sont pas indiqués intrinsèquement, tandis que dessiner un oiseau, courir un cent mètres, chanter une chanson, boire un verre d'eau, fumer une cigarette, manger un gâteau, écrire une lettre sont des accomplissements : le début et la fin de ces procès sont délimités par les compléments nominaux.

(9) a. ?? Je suis en train de dessiner dix oiseaux.

b. Je suis en train de dessiner les dix oiseaux que je t'avais promis.

Lorsque les compléments d'objet sont supérieurs à une unité, comme dans (9a), il nous paraît très difficile de recourir à en train de (cf. Franckel, 1989 : 69). S'agissant de noms comptables (oiseaux, chansons, verres d'eau, cigarettes, gâteaux, lettres...), on ne peut pas, en même temps, être en train de dessiner dix oiseaux, chanter dix chansons, boire dix verres d'eau, fumer dix cigarettes, manger dix gâteaux, écrire dix lettres, etc. En revanche, (9b) peut être accepté en ce sens que son prédicat est représenté mentalement comme un bloc à exécuter dans son ensemble, où le caractère comptable du complément oiseau n'est pas pris en compte.

\section{c. Les achèvements}

Un achèvement est un «changement atomique »(Gosselin, ibid.). Bien que les achèvements soient des procès dynamiques comme des activités et des accomplissements, ils ne sont pas compatibles, en théorie, avec en train de. Cette incompatibilité est due au fait que les achèvements désignent un changement « ponctuel », «momentané » ou «atomique », alors que cette périphrase, «indicateur de processus », exige logiquement une certaine durée.

(10) Paul est en train de trouver une solution.

(11) Paul est en train d'atteindre le sommet.

(12) Paul est en train de gagner (perdre).

Toutefois, le choix de être en train de avec les achèvements n'est pas exclu. Leur conflit pouvant être neutralisé nécessite une réinterprétation : les achèvements sont donc transformés en accomplissements. Dans (10 à 12), on décrit la phase préparatoire qui demande une certaine durée, et non la phase ultime, synonyme d'un changement «atomique ». Dans (10), Paul est sur une bonne voie : la solution est imminente. Dans (11), Paul se rapproche du sommet. Dans (12), le locuteur constate qu'au cours du commentaire en direct d'un jeu, Paul se trouve dans une position très favorable pour gagner ou au contraire très défavorable avec le risque de perdre.

(13) a. Je déclare ouverts les Jeux Olympiques.

b. * Je suis en train de déclarer ouverts les Jeux Olympiques.

c. Il est en train de déclarer ouverts les Jeux Olympiques.

Dans l'énoncé performatif (13a) à procès perfectif de type achèvement, l'énonciateur $(\mathrm{J} e=\mathrm{le}$ Président de la République, par exemple) accomplit l'action de déclarer au moment où il la prononce à $\mathrm{t}^{\circ}$. L'emploi du présent est obligatoire, ce qui exclut le choix de être en train de, comme dans (13b). En revanche, son recours est envisageable dans l'énoncé constatif (13c), lorsqu'on veut décrire le moment où le Président (Il) est en train de déclarer. A la grande différence de (13a), on peut donner une réponse positive (vrai) ou négative (faux) à (13c).

\section{d. Les états ${ }^{13}$}

L'état nécessaire est une «situation stable sans début (changement initial) ni fin (changement final)», alors que l'état contingent est une «situation stable avec début et fin (d'où la compatibilité des prédicats exprimant ce type de procès avec commencer à et cesser de). Pour

13 Selon Fuchs, Gosselin et Victorri (1991 : 148), les états sont subdivisés en quatre sous-groupes. Les propriétés nécessaires sont être mortel, avoir un angle droit... Les propriétés permanentes sont exprimées par être rouge, avoir les yeux bleus, être vrai... Les propriétés contingentes stables sont représentées par être coléreux, être gentil, être méchant... Les propriétés contingentes instables sont traduites par être en colère, être fatigué, être malade... 
autant le début et la fin ne sont pas perçus comme appartenant au procès lui-même, mais comme des limitations extrinsèques »(Gosselin, op. cit. : 54).

(14) * L'homme est en train d'être mortel.

(15) * Paul est en train d'avoir des yeux bleus.

Puisque en train de contient le trait dynamique, elle n'est donc pas en principe compatible avec les procès qui en sont dépourvus, à savoir les états. Si l'incompatibilité est totale entre cette périphrase et l'état nécessaire et l'état permanent, comme dans (14 et 15), elle ne l'est pas tout à fait à l'égard des états contigents à propriétés stables ou instables. Leur combinaison, n'étant pas exclue, nécessite toutefois une réinterprétation. Observons maintenant les quelques énoncés suivants comprenant le verbe d'état aimer et en train de :

(16) a. Ils s'aiment.

b. Ils sont en train de s'aimer.

(17) Jean est en train d'aimer Marie (Fuchs et Léonard, 1979 : 331)

(18) Jean aime vite et il est en train d'aimer à nouveau (Wilmet, $1980: 61$ )

(19) Il faut toujours que tu sois en train d'aimer.

Le procès s'aimer, qui est un état contingent stable signifiant «éprouver une affection réciproque », est en principe compatible avec le présent, comme dans (16a). Mais le recours à en train de, n'étant pas impossible, transforme en quelque sorte ce procès statif en activité ; s'aimer pourrait vouloir dire aussi «avoir des relations sexuelles »: (16b) signifie qu'ils sont en train de faire l'amour.

Selon Fuchs et Léonard (ibid.), la combinaison de en train de avec aimer dans (17) doit être comprise comme suit : «Jean commence à éprouver une affection pour Marie ». Dans (18), l'adverbe vite, permettant une lecture itérative du procès aimer (soulignée plus loin par à nouveau), rend celui-ci dynamique : Jean aime vite signifie donc « Jean tombe vite amoureux ou Jean est amoureux rapidement » (cf. Wilmet, ibid.). Par conséquent, l'emploi de être en train de est possible pour souligner le déroulement du procès. Dans (19), à partir d'un cas concret, répété à plusieurs reprises, le locuteur cherche à généraliser le comportement de son allocutaire, encore amoureux. Autrement dit, l'allocutaire est en humeur d'aimer, et cet état, souligné par « toujours », l'habite en permanence.

Notons que (18 et 19) se ressemblent en ce que le verbe aimer, sans le complément d'objet direct, est en emploi absolu, à la différence de l'emploi d'aimer dans (17). Précisons en outre que les trois exemples $(17,18$ et 19), à la différence de (16b), n'ont pas le sens de «avoir des relations sexuelles ».

(20) a. Paul est en train de se montrer méchant.

b. Paul est en train de se montrer trop gentil.

Les procès être méchant et être gentil sont des états à propriétés stables, qui ne sont pas en principe compatibles avec en train de. Cependant, dans (20a et b), le locuteur ne décrit pas le caractère général de Paul, mais son comportement au moment de leur rencontre. C'est l'emploi $\mathrm{du}$ verbe se montrer qui permet de conclure qu'il s'agit d'une situation occurrencielle qui se déroule à $\mathrm{t}^{\circ}$. L'adjectif gentil, suivi de trop, a une valeur dépréciative, tout comme l'adjectif méchant. Dans (20b), le locuteur pense peut-être que ce comportement « gentil » cache quelque chose. Nous partageons le point de vue de Franckel (1989), selon lequel en train de peut être compatible avec les états, mais plus précisément avec les états qui représentent des valeurs dépréciatives, comme dans (4 à 7).

\section{2. Être en train de avec déjà, encore, toujours et en ce moment}

Nous procéderons ici à une étude de compatibilité sémantique, d'une part entre être en train de et déjà, encore, toujours, d'autre part entre cette périphrase et en ce moment.

a. Être en train de avec déjà, encore et toujours 
Selon Hœpelman et Rohrer (1980 : 125-128) et Fuchs, Gosselin et Victorri (1991 : 137), Grevisse (1993, §967), déjà, encore et toujours ont en commun les valeurs durative et répétitive. Puisque la périphrase être en train de est un «indicateur de processus », qui exige une certaine durée, elle doit être compatible sémantiquement avec ces adverbes, lorsqu'ils n'expriment que la valeur durative. Examinons les exemples suivants, dont les procès sont des accomplissements :

(21) Paul est déjà en train de dessiner le plan de sa maison.

(22) Paul est encore en train de dessiner le plan de sa maison.

(23) Paul est toujours en train de dessiner le plan de sa maison.

A la différence de (22 et 23), (21) avec déjà souligne que Paul a commencé à dessiner le plan plus tôt que prévu, ou tout au moins qu'il a entrepris ce travail sans perdre de temps. (22) avec encore indique que la tâche de Paul n'est pas encore terminée, alors que (23) avec toujours diffère légèrement de (22) : toujours souligne la permanence ou la persistance du procès à $t^{\circ}$.

\section{b. Être en train de avec en ce moment}

Lorsque le point de référence du procès est simultané à $t^{\circ}$, on a tendance à utiliser le présent suivi de en ce moment, ce qui peut se vérifier surtout dans des énoncés décrivant des phénomènes météorologiques. Le recours à cette locution adverbiale ne s'est-il pas plié à l'art du bien dire imposé autrefois par les grammairiens puristes, selon qui il fallait dire Il écrit en ce moment et non Il est après écrire, ou à écrire, etc. N'oublions pas que la structure être en train de fut vivement critiquée par certains grammairiens du $\mathrm{XIX}^{\mathrm{e}}$ siècle $^{14}$, avant d'être adoptée ultérieurement.

(24) a. Il est en train de pleuvoir (neiger, venter...).

b. Il pleut (neige, vente...) en ce moment.

c. La pluie (la neige) est en train de tomber / Le vent est en train de se lever.

d. Il pleut souvent à Londres.

Nous notons que en train de s'emploie moins avec les énoncés impersonnels indiquant des phénomènes météorologiques comme dans (24a), et que (24b) avec en ce moment nous semble plus naturel. Selon Franckel (op. cit. : 84), «Il pleut s'emploie de façon beaucoup moins contrainte que Il est en train de pleuvoir ». Si on veut recourir à cette périphrase, il convient de faire appel à (24c), qui nous paraît naturel.

(25) a. It's raining (snowing...)

b. It often rains in London.

En français, lorsqu'on décrit un procès en cours ou un procès habituel, on utilise le présent simple, suivi de en ce moment dans (24b), ou de souvent dans (24d). En anglais, on doit se servir de la forme progressive -ing dans (25a) et du présent simple dans (25b).

John is singing.

Jean chante, en ce moment (Desclés, op. cit. : 187).

Il est intéressant de constater que dans (26), l'auteur a traduit la forme progressive anglaise non par en train de, mais par en ce moment, alors que, ne s'agissant pas d'un énoncé décrivant un phénomène météorologique, on peut très bien user de cette périphrase française. Cela amène à penser que -ing en anglais est une flexion, et que son processus de grammaticalisation est plus avancé et plus stable que celui de en train de (cf. Pusch, op. cit., déjà évoqué à \$1.5b).

(27) As-tu fait tes devoirs?

a. Non, je suis en train (de les faire).

b. Non, je les fais en ce moment.

c. ?Non je les fais.

14 «En 1845, Francis Wey, dans ses Remarques sur la langue française..., condamne cette locution de la façon la plus formelle, comme un parisianisme vulgaire et provincialisme de la région de Chartres » (Gougenheim, op. cit. : 64). 
À une question ouverte (27), on peut obtenir les réponses possibles suivantes: Oui, je les ai faits, ou Non, je suis en train (de les faire) dans (27a), qui nous semble plus naturel que (27b). Il est plus difficile de recourir à $(27 \mathrm{c})$.

(28) a. Je suis en train de manger, c'est alors qu'il prend place près de moi et se met à me raconter son histoire...

b. *Je mange en ce moment, c'est alors qu'il prend place près de moi [...]

c. ??Je mange, c'est alors qu'il prend place près de moi [...]

Dans le contexte de (28a), le choix du présent narratif avec être en train de permet de souligner une action ponctuelle (prendre place) qui vient interrompre une autre en train de progresser (manger). En revanche, il ne nous semble pas possible de recourir à en ce moment dans $(28 b)$ à cause de l'incompatibilité entre ce circonstanciel de temps qui réfère à $t^{\circ}$ et le présent narratif racontant un fait passé. Quant à (28c), il est beaucoup moins naturel que (28a).

\section{3. Être en train de avec les formes simples et les formes composées de l'indicatif}

\section{a. Être en train de avec les formes simples}

Notre étude sera limitée à la combinaison de être en train de avec les formes simples (le présent, l'imparfait, le passé simple, et le futur), et avec les formes composées du mode indicatif (le passé composé, le plus-que-parfait, le passé antérieur, et le futur antérieur). En revanche, nous n'examinons ici ni le conditionnel présent (forme simple) ni le conditionnel passé (forme composée).

(29) Je suis en train de faire des vocalises.

(30) Hier, j'étais en train de faire des vocalises lorsque Paul est venu.

(31) * Je fus en train de faire des vocalises.

(32) Demain, je serai en train de faire des vocalises lorsque Paul viendra me rejoindre pour la répétition de notre concert.

Le présent et l'imparfait sont tout à fait compatibles avec en train de comme en témoignent les exemples (29 et 30). Quant au futur simple, il peut se combiner aussi avec en train de, comme dans (32).

En revanche, le passé simple dans (31), bien qu'il soit une forme simple, n'est pas compatible avec cette périphrase, comme l'a fait remarquer Both-Diez (1985 : 14, cité par Leeman, 2003 : 30) : «La locution prépositive être en train de, qui marque l'action en cours, l'aspect duratif du verbe, ne s'accommode ni du passé simple, ni du passé composé ».

Dans (31), qui est un énoncé simple, le passé simple ne peut pas s'associer avec en train de, à cause de leur incompatibilité aspectuelle : tandis que cette périphrase indique « une focalisation sur un processus pris en cours d'accomplissement, donc qui a une certaine durée, sans se prononcer sur son début ou sa fin » (Leeman, op. cit. : 30), le passé simple, quant à lui, traduit l'aspect global en ce sens que ce temps « donne du procès une vision globale, c'est-à-dire qu'il en présente tout à la fois le terme initial, le développement complet, et le terme final. Le procès est perçu dans sa globalité comme une totalité finie » (Denis et Sancier-Château, 1994 : 277).

Toutefois, Wilmet (op. cit. : 59), Franckel (op. cit. : 76) confirment que en train de n'est compatible avec le passé simple que lorsque ce temps est utilisé dans une subordination : «la composition être en train de + infinitif [...] pallie l'absence d'un aspect sécant (Quand il fut en train de chanter...) » (cf. Wilmet).

(33) Quand le nain Bibobu fut bien en train de dormir, Mitou et Miquette sortirent sans faire aucun bruit (Franckel, op. cit. : 76).

(33a) Quand le nain Bibobu fut bien en train de ranger sa maison, Mitou et Miquette sortirent sans faire aucun bruit (exemple modifié). 
Si la combinaison entre en train de et le passé simple est possible, comme dans (33), c'est parce que cet énoncé complexe permet de souligner une opposition aspectuelle entre l'aspect progressif représenté par en train de, suivi du procès de type activité (dormir), qui exige une certaine durée (la durée du sommeil de Bibobu), et l'aspect global, indiqué par le procès de type achèvement (sortir) au passé simple en proposition principale. Précisons que la construction quand - passé simple - passé simple dans (33) n'a pas de valeur répétitive (à chaque fois que), mais qu'il s'agit d'un fait unique. Il nous paraît possible de remplacer le procès de type activité (dormir) dans (33) par le procès de type accomplissement (ranger sa maison) dans (33a).

Nous estimons que les éléments constitutifs de la subordination de (33) sont tout à fait compatibles entre eux pour les raisons suivantes : la conjonction quand, qui institue « un point de départ» (cf. Leeman, op. cit. : 31), est compatible avec le passé simple, dont «l'effet de sens typique est celui du passé simple aoristique [...] inchoatif, situant le procès (au moins son début) dans le passé non immédiat [...] » (cf. Gosselin, op. cit.: 197). En outre, en train de permet de saisir le procès en son début (phase initiale). L'emploi de bien, pertinent, vise à insister sur le début de la phase «bien dormir » en séparant celle-ci de la phase précédente «pas encore bien dormir ». En résumé, le choix de quand, du passé simple, de la périphrase en train de, et de bien met clairement l'accent sur l'aspect inchoatif. En d'autres termes, pour sortir, Mitou et Miquette attendent le moment où la phase « bien dormir » commence.

Nous partageons les remarques pertinentes de Leeman (ibid.), qui souligne que la combinaison entre en train de et le passé simple ne s'avère possible que lorsque les procès sont saisis à leur début ou à leur terme, et que cela dépend de la nature des conjonctions. En effet, l'auteur en distingue deux types : d'une part, quand, dès que, (aus)sitôt que, qui «instituent un point de départ », et tant que, tout le temps que, aussi longtemps que «qui impliquent une vision globale, incluant la borne de fin », d'autre part, tandis que, alors que, depuis que, pendant que, qui «supposent une saisie sécante ». Selon Leeman, le premier type de conjonctions (quand, dès que, (aus)sitôt que, tant que, tout le temps que, aussi longtemps que) est compatible avec le passé simple, alors que le second type de conjonctions (tandis que, alors que, depuis que, pendant que) ne l'est pas.

A partir des observations de Leeman, nous dressons le tableau récapitulatifs ci-dessous relatif aux résultats de combinaison entre en train de et le passé simple en subordination.

\begin{tabular}{|c|c|c|c|}
\hline Types de conjonctions & Temps verbaux & Périphrase & Résultats de la combinaison \\
\hline $\begin{array}{l}\text { quand, dès que, } \\
\text { aus(sitôt) que }\end{array}$ & passé simple & en train de & [+ compatible $]$ \\
\hline $\begin{array}{l}\text { tant que, tout le temps } \\
\text { que, aussi longtemps que }\end{array}$ & passé simple & en train de & [+ compatible] \\
\hline \multirow{2}{*}{$\begin{array}{l}\text { pendant que, tandis que, } \\
\text { alors que, depuis que }\end{array}$} & passé simple & \multirow{2}{*}{ en train de } & [- compatible] \\
\hline & imparfait & & [+ compatible] \\
\hline
\end{tabular}

Nous constatons que, dans un énoncé complexe, la relation de compatibilité entre les types de conjonctions et les temps verbaux choisis l'emporte sur celle entre les types de conjonctions et en train de. En effet, cette périphrase peut se combiner avec toutes les conjonctions figurant dans le tableau, sauf toutefois lorsque le passé simple est utilisé dans les énoncés complexes introduits par tandis que, alors que, depuis que, pendant que, le résultat de la combinaison est alors négatif. Il suffit de remplacer le passé simple par l'imparfait pour que ces conjonctions soient compatibles avec cette périphrase.

\section{b. Être en train de avec les formes composées}

(34) *J'ai été en train de faire des vocalises.

(35) * J'avais été en train de faire des vocalises. 
* J'eus été en train de faire des vocalises.

* J'aurai été en train de faire des vocalises.

Selon Franckel (op. cit. : 69) et Franckel et Paillard (1991 : 133), être en train de et le passé composé sont « strictement incompatibles entre eux », pour la raison suivante : «Il a été en train de $P$ marque donc une contradiction entre la fermeture imposée par le passé composé et l'ouverture imposée par être en train de » (Franckel, ibid.). Ces explications peuvent s'appliquer aussi aux autres formes composées (34 à 37). Toutefois, les exemples suivants illustrent l'association du plus-que-parfait et du passé composé avec cette périphrase :

(38) J'y sonnai comme au feu. J'y retentis comme une trompette, comme si l'ennemi avait été en train d'enlever le drapeau du régiment! (Barbey D'Aurevilly, Les Diaboliques, Le Drapeau cramoisi, p. 82, Frantext, cité par Havu, 2006 : 57).

En général, après la conjonction comme si, on recourt à l'imparfait ou au plus-que-parfait (cf. Grevisse, op. cit. : 1672) pour exprimer une comparaison reposant sur une supposition. L'emploi de l'imparfait ou du plus-que-parfait dépend de la nature des procès : les procès imperfectifs sont à l'imparfait, alors que les procès perfectifs sont au plus-que-parfait. Dans (38), le plus-que-parfait indique que le procès perfectif (enlever le drapeau) est accompli. La combinaison entre ce temps verbal et en train de n'est pas possible dans une phrase simple, comme dans (35), mais est envisageable dans une subordination introduite par comme si.

(39) Il y a eu des périodes où ils ont été en train de se battre, il y a eu des périodes où ils ont été copain-copain pendant des périodes courtes [...] (internet, traduction française de l'interview de Joe Ybarra de la société Cheyenn Mountain Entertainment, qui a eu lieu lors du salon de l'E3 à Los Angeles, cité par Havu, ibid.).

Le passé composé dans (39) et le plus-que-parfait dans (38) sont situés tous les deux dans une subordination. Il existe donc une contrainte syntaxique relative à l'emploi des formes composées avec en train de. Cette contrainte s'est déjà vérifiée avec le passé simple (cf. Leeman).

(40) CECODEL a déjà six années d'existence [...]. Pendant ces six années de notre existence, nous avons été en train de cimenter notre organisation en déployant de grands efforts dans plusieurs sens: travaux d'identification [...]. (www.cecodel. org/presid_fr.htm, 23/03/2005, K. M. El Ouariachi, cité par Havu, ibid.).

Dans (40), pendant, suivie d'une indication temporelle précise (six années), exclut l'imparfait: "Pendant ces six années..., nous *étions en train de cimenter... », et favorise plutôt le passé composé comme suit : "Pendant ces six années..., nous avons cimenté... ». Dans cet exemple, l'auteur cherche à faire coexister le passé composé et en train de pour mettre l'accent sur l'aspect accompli et l'aspect progressif, ce qui risque de susciter un rapport de conflit entre ces deux aspects, comme l'a souligné Franckel (op. cit. : 69).

Havu (ibid.) souligne qu' on rencontre sur internet, à l'aide du moteur de recherche Google, « un nombre non négligeable d'exemples » illustrant la combinaison entre en train de et des formes composées comme le passé composé, le plus-que-parfait, etc. L'auteur tente d'expliquer ce phénomène comme suit : "il semble que la contruction être en train de + inf soit susceptible d'acquérir une valeur actionnelle dans le français contemporain, surtout dans certains registres informels. C'est ce qui est arrivé en espagnol (he estado escribiendo; había estado escribiendo, et en anglais (I have been writing ; I had been writing, etc.)».

Doit-on considérer l'association du passé composé ou du plus-que-parfait avec en train de comme une faute ou un calque de l'anglais ou de l'espagnol?

\section{CONCLUSION}

Le processus de grammaticalisation du substantif train, combiné au verbe être et aux prépositions en et de pour former progressivement être en train et être en train de, a duré environ sept siècles, du $\mathrm{XII}^{\mathrm{e}}$ au $\mathrm{XIX}^{\mathrm{e}}$ siècle. Quant à l'expression être en train de, nous avons 
noté une évolution sémantique en ce sens qu'elle exprimait, au XVII ${ }^{\mathrm{e}}$ siècle, l'imminence de l'exécution d'une action, «être disposé à », et qu'elle traduit, de nos jours, l'aspect progressif, pouvant commuter, dans certains contextes, avec être en plein au sens de «au milieu de ». Parallèlement, être en train de, qui était encore compatible jusqu'au milieu du XIX ${ }^{\mathrm{e}}$ siècle aussi bien avec des noms qu'avec des verbes, est devenue aujourd'hui un véritable auxiliaire d'aspect verbal : la périphrase n'accepte plus que des verbes.

Le trait [+ dynamique] contenu dans le substantif train et aussi dans ses locutions dérivées a permis d'expliquer pourquoi être en train de, grammaticalisée, devient un «indice de processus » parfaitement compatible avec les procès dynamiques pourvus d'une certaine durée, à savoir les activités et les accomplissements. La combinaison de cette périphrase avec les achèvements, ponctuels, n'étant pas exclue, conduit à transformer ceux-ci en accomplissements. Autrement dit, le locuteur décrit la phase préparatoire avant la phase ultime. C'est probablement à cause de son trait [+ dynamique] qu'en théorie être en train de n'est pas compatible avec les états nécessaires ou permanents [- dynamique]. Toutefois, l'association de cette périphrase et de certains états stables, n'étant pas impossible, nécessite des explications implicant le recours au sens modal «être en humeur de ».

Le fait que l'aspect progressif ne soit traduit en français de nos jours que par des périphrases (en train de, en cours de, en voie de, etc.), et non par une morphologie flexionnelle comme -ing en anglais, montre que ces périphrases françaises sont moins grammaticalisées que la forme anglaise.

Danh Thành DO-HURINVILLE

dhdthanh@gmail.com 